\begin{tabular}{|c|c|}
\hline Title & Formation of motile assembly of microtubules driven by kinesins \\
\hline Author(s) & Kawamura, Ryuzo; Kakugo, A kira; Shikinaka, Kazuhiro; Osada, Y oshihito; Gong, Jian Ping \\
\hline Citation & $\begin{array}{l}\text { Smart Materials sand Structures, } 20(12), 124007 \\
\text { https://doi .org/10.1088/0964-1726/20/12/124007 }\end{array}$ \\
\hline Issue Date & $2011-12$ \\
\hline Doc URL & http:/hdl.handle.net/2115/48144 \\
\hline Rights & $\begin{array}{l}\text { This is an author-created, un-copyedited version of an article accepted for publication in Smart Materials and Structures. } \\
\text { IOP Publishing Ltd is not responsible for any errors or omissions in this version of the manuscript or any version } \\
\text { derived from it. The definitive publisher authenticated version is available online at } 10.1088 / 0964-1726 / 20 / 12 / 124007 \text {. }\end{array}$ \\
\hline Type & article (author version) \\
\hline File Information & SMS20-12_124007.pdf \\
\hline
\end{tabular}

Instructions for use 


\section{Formation of motile assembly of microtubules driven by kinesins}

Ryuzo Kawamura $^{1 \dagger}$, Akira Kakugo ${ }^{1,2}$, Kazuhiro Shikinaka ${ }^{1 \ddagger}$, Yoshihito Osada ${ }^{1 \dagger}$, and Jian Ping Gong $^{1 *}$

${ }^{1}$ Division of Biological Sciences, Graduate School of Science, Hokkaido University, Sapporo 060-0810, Japan

${ }^{2}$ PRESTO, Japan Science and Technology Agency, 4-1-8 Honcho, Kawaguchi, Saitama 332-0012, Japan

3

${ }^{\dagger}$ Present address: RIKEN, 2-1, Hirosawa, Wako, Saitama 351-0198, Japan.

${ }^{\ddagger}$ Present address: Institute of Symbiotic Science and Technology, Tokyo University of Agriculture and Technology, Tokyo 184-8588, Japan.

*Author to whom correspondence should be addressed. E-mail: gong@sci.hokudai.ac.jp (J.P.G.)

Telephone/FAX: +81-11-706-2774

\section{Abstract}

Microtubule (MT) and kinesin are rail and motor proteins that are involved in various moving events of eukaryotic cells in natural systems. In vitro, the sliding motion of microtubules (rail) can be reproduced on a kinesin (motor protein)-coated surface coupled with adenosine triphosphate (ATP) hydrolysis, which is called a "motility assay". Based on this technique, a method was recently established to form MT assemblies by an active self-organization (AcSA) process, in which MTs are crosslinked during a sliding motion on a kinesin-coated surface. Streptavidin (ST) was employed as glue to crosslink biotin-labeled MTs. Various shapes, sizes, and motilities were formed with the AcSA MT assemblies, depending on the initial conditions. In this paper, we briefly review our recent work on the formation of MT assemblies on a kinesin-coated surface. 


\section{Introduction}

As power units, biological actuators of living organisms have attractive features such as high-energy efficiency, flexibility, and a wide range of sizes that can be seen from bacterial flagella to muscles of animals, which differ from the power units of conventional man-made machines [ref. 1, 2]. These complex functionalities are accomplished by integrating, synchronizing, and controlling the nano-metric motion of motor proteins into hierarchical structures. However, in vitro reproduction of these high-ordered hierarchical structures remains challenging. In artificial systems, the motor protein mechanisms are progressively being elucidated at the molecular level for several motor proteins such as $F_{1} F_{0} /$ ATPase, actin-myosin, and MT-kinesin systems [ref. 3-5]. Recently, investigation of motor protein mechanisms of action has been expanded to include several multi-molecular systems [ref. 6-9]. We have also investigated the integration of these motor proteins that possess their "moving" property with an aim to show the motility of motor proteins as a larger actuator than nano-devices, which is addressed using motor proteins at the single molecular level.

Motor proteins can be categorized by their motions into two groups: rotating motors and linear motors [ref. 10]. The well-known rotating motors are bacterial flagellum and the mitochondrial $F_{1} F_{0}$-ATPase, which is driven by ATP hydrolysis. The linear motor protein systems actin-myosin and MT-kinesin have been widely studied and are involved in the control of movement in larger living organisms, including the organs of animals. In contrast, rotary motors are involved in small movement in mitochondria and smaller organisms such as bacteria. As such, linear motor proteins are promising systems to model multi-molecular integrated structures to investigate larger motions using linear rails that can interact with multiple motors. Actin-myosin or MT-kinesin (or dynein), which are well-known linear motor systems, has been proposed as the building blocks of ATP-fueled biomachines [ref. 7, 11-18]. In previous papers, we reported that large linear actin bundles show sliding motions on a myosin-coated surface in the presence of ATP. Actin bundles, which consist of several tens of filamentous actin, can be obtained through electrostatic interactions with synthetic polymers carrying positive charges [ref. 19-23]. The MT-kinesin system has also been used to form assemblies with unique properties. Recently, a method was developed to integrate MTs into bundle structures by crosslinking moving MTs on a kinesin-coated surface under the presence of adenosine triphosphate (ATP); MTs were covalently modified with biotins, which were partially covered with streptavidin (ST), such that two MTs could interact and form a bundle (Figure 2a) [ref. 24]. In previous studies, we studied this system by systematically investigating the effects of various parameters such as the concentration of the kinesin "motor," ST "glue," and MT "assembly component," represented as $C_{k}, C_{S T}$, and $C_{\text {Tubr }}$ 
respectively, on the formation and motility of MT bundles. Although the assemblies of MTs are much more primitive compared to the hierarchical structure of actuators in natural systems, the process to form assemblies of motor protein systems is critical to mimic and study the natural system. This survey on MT assembly formation provides information to aid in the application of motor proteins, including the MT-kinesin and actin-myosin systems, as building blocks of ATP-fueled biomachines.

\section{Abbreviations}

$\mathrm{C}_{\mathrm{k}}$ : concentration of kinesin $[\mathrm{nM}]$

$\mathrm{C}_{\mathrm{ST}}$ : concentration of streptavidin $[\mathrm{nM}]$

$\mathrm{C}_{\text {Tub }}$ : concentration of MT indicated by the amount of tubulin [nM]

MT: microtubule

MAPs: microtubule-associated proteins

GTP: guanosine-5-triphosphate

GFP: green fluorescent protein

DMSO: dimethyl sulfoxide

GMPCPP: guanylyl-(a, $\beta)$-methylene-diphosphonate 


\section{Experimental setup and analysis}

\subsection{Preparation of tubulins and MTs}

Traditionally, tubulin has been purified from porcine brain since the early 1970 s by using ion-exchange chromatography [ref. 25, 26]. In the tubulin purification process, removal of microtubule-associated proteins (MAPs) is important, since their contamination strongly affects the polymerization of tubulins. MAPs can be removed from tubulins by using high-salt concentrations, which is a simplified method to obtain pure and highly active tubulins [ref. 27, 28]. Currently, tubulin and tubulins labeled with fluorescent probes are now commercially available.

In our experiments, tubulin was purified from porcine brain by using a high-concentration PIPES buffer [ref. 28]. A tubulin aliquot was modified with biotins to crosslink MTs to each other by streptavidin; streptavidin has four biotin-interacting sites with a very high binding constant for biotin $\left(K_{D}=\sim 10^{-14}\right)$ [ref. 29]. Lysine residues on the MT surface were modified with biotin-XX-SE (Invitrogen) according to standard techniques [ref. 30]. The labeling stoichiometry was approximately 2 biotins per tubulin, which was estimated by spectrometric titration using the 2-(4'-hydroxyphenylazo)benzoic acid (HABA) dye (Dojindo) [ref. 31]. Another aliquot of tubulins was fluorescently labeled to observe MTs by fluorescent microscopy. Tetramethylrhodamine succinimidyl ester (TAMRA-SE) (Invitrogen) was chemically crosslinked to the lysine residues of tubulin according to standard techniques [ref. 32]. The labeling ratio was 1.2 , as determined by measuring the absorbance of the protein and tetramethylrhodamine at $280 \mathrm{~nm}$ and $555 \mathrm{~nm}$, respectively.

\subsection{Preparation of kinesin}

Kinesins can also be purified from porcine brain by using a traditional method [ref. 33-35]. Alternatively, recombinant kinesins can be purified from an $E$. coli expression system, which is in contrast to tubulins that cannot polymerize to MTs when the recombinant protein was prepared from an E. coli expression system [ref. 36]. For our studies, the first 560 amino acids of kinesin-1 were fused to the green fluorescent protein (K560-GFP), as described earlier by partially modifying the expression and purification methods [ref. 37]. The GFP moiety is designed to effectively immobilize K560-GFP to a glass surface by binding to anti-GFP antibodies. 


\subsection{Microtubule polymerization}

MTs for the in vitro motility assay were prepared by polymerizing tubulins with guanosine-5'-triphosphate (GTP) and stabilizing with paclitaxel, which is a MT-stabilizing agent [ref. 38].

To obtain biotin- and rhodamine-labeled MTs, the mixture of biotin-tubulin, rhodamine-tubulin, and native tubulin (molar ratio of 45:10:45; final concentration was $24 \mu \mathrm{M}$ ) was used in the MT polymerization procedure. Based on the labeling ratio of biotin and rhodamine to tubulins, the final labeling ratio was calculated as 0.9 and 0.1 to tubulin $[\mathrm{mol} / \mathrm{mol}]$ for biotin and rhodamine, respectively.

In our experiments, MTs were polymerized using two different procedures. The first was a standard method in which tubulin $(24 \mu \mathrm{M})$ was polymerized in a polymerization buffer (80 mM PIPES-2K, $1 \mathrm{mM}$ EGTA, $5 \mathrm{mM} \mathrm{MgCl}$, $1 \mathrm{mM} \mathrm{GTP}$, and 5\% DMSO; pH adjusted to 6.8 with $\mathrm{HCl}$ ) at $37^{\circ} \mathrm{C}$ for $30 \mathrm{~min}$. The solution containing the MTs was then diluted 50 - or 100-fold with a stabilizing buffer (80 mM PIPES-2K, $1 \mathrm{mM} \mathrm{EGTA,} 1 \mathrm{mM} \mathrm{MgCl} 2,1 \%$ DMSO, and $10 \mu \mathrm{M}$ paclitaxel; $\mathrm{pH}$ adjusted to 6.8 with $\mathrm{HCl}$ ) and gently pipetted 10 times. Hereafter, $C_{\text {Tub }}$ represents the concentration of tubulins. The concentration of diluted MTs was 480 or 240 $\mathrm{nM}$. Diluted MTs were incubated at $\sim 25^{\circ} \mathrm{C}$ until the preparations for the motility assays were complete, which generally took 0-24 h.

The second method of MT polymerization was used to obtain rigid MTs using guanylyl-(a, $\beta)$-methylene-diphosphonate (GMPCPP), which is a non-hydrolyzable GTP analogue, by using a reported method [ref. 39, 40]. In this procedure, tubulin $(40 \mu \mathrm{M})$ was incubated in a GMPCPP-polymerization buffer that contains $1 \mathrm{mM}$ GMPCPP (Jena Bioscience, Jena, Germany), instead of GTP, at $37^{\circ} \mathrm{C}$ for $10 \mathrm{~min}$. Then, the polymerized MTs, hereafter called "seed," were diluted 1000-fold in an elongation buffer containing fresh tubulins $(1 \mu \mathrm{M})$ in the GMPCPP-polymerization buffer. The solution, which contained a total of $1.04 \mu \mathrm{M}$ tubulin, was incubated for $4 \mathrm{~h}$ at $37^{\circ} \mathrm{C}$. Thereafter, the elongated MTs were diluted to $480 \mathrm{nM}$, which is indicated as the tubulin concentration, and were stored at room temperature until used for the MT motility assay. In this study, MTs polymerized with GMPCPP are called GMPCPP-MTs and those polymerized with GTP and stabilized with paclitaxel are called GTP-MTs. 


\subsection{Motility assay setup and parameters}

Flow cells were prepared by placing a cover glass on a slide glass equipped with a pair of spacers to form a chamber of approximate dimensions $4 \times 18 \times 0.1 \mathrm{~mm}^{3}(W \times L \times H)$. A single layer of parafilm was used to fix the spacer-separated glasses by heating. The flow cell was filled with $0.2 \mathrm{mg} \mathrm{ml}^{-1}$ anti-GFP antibody (Invitrogen) for $15 \mathrm{~min}$, and then washed with 48 $\mu \mathrm{l}$ of casein solution (80 mM PIPES-2K, $1 \mathrm{mM}$ EGTA, $1 \mathrm{mM} \mathrm{MgCl}{ }_{2}$, and $\sim 0.5 \mathrm{mg} \mathrm{ml}^{-1}$ casein; $\mathrm{pH}$ adjusted to 6.8 with $\mathrm{HCl}$ ). After incubating with the casein solution for $5 \mathrm{~min}$ to block the antibody-unbound glass surface, $24 \mu \mathrm{l}$ of the K560-GFP solution (0.63-63 nM in kinesin solution; 80 mM PIPES-2K, 40 mM NaCl, $1 \mathrm{mM} \mathrm{EGTA,} 1 \mathrm{mM} \mathrm{MgCl} 2,0.5 \mathrm{mg} \mathrm{ml}^{-1}$ casein, 1 $\mathrm{mM}$ DTT, $4.5 \mathrm{mg} \mathrm{ml}^{-1} \mathrm{D}$-glucose, $50 \mathrm{U} \mathrm{ml}^{-1} \mathrm{glucose}$ oxidase, $50 \mathrm{U} \mathrm{ml}^{-1}$ catalase, $10 \mu \mathrm{M}$ paclitaxel, and $1 \%$ DMSO; $\mathrm{pH} 6.8$ ) was introduced, and this mixture was incubated for 5 min to allow kinesins to bind to the antibody. Hereafter, $C_{k}$ represents the kinesin concentration. The flow cell was washed with $32 \mu$ of motility buffer ( $80 \mathrm{mM}$ PIPES-2K, $1 \mathrm{mM} \mathrm{EGTA,} 2 \mathrm{mM} \mathrm{MgCl}$, $0.5 \mathrm{mg} \mathrm{ml}^{-1}$ casein, $1 \mathrm{mM}$ DTT, $4.5 \mathrm{mg} \mathrm{ml}^{-1} \mathrm{D}$-glucose, $50 \mathrm{U} \mathrm{ml}^{-1}$ glucose oxidase, $50 \mathrm{U} \mathrm{ml}^{-1}$ catalase, $10 \mu \mathrm{M}$ paclitaxel, and $\sim 1 \%$ DMSO; pH 6.8). A diluted solution ( $24 \mu \mathrm{l}$ ) of $9.6 \mathrm{nM}$ MTs in motility buffer was added and incubated for $5 \mathrm{~min}$, and then washed with $32 \mu \mathrm{l}$ of motility buffer. Biotins on the MT surface were partially covered with streptavidin-FITC (ST) (Wako) by incubating biotin with the ST solution $(0-4800 \mathrm{nM}$, which is equimolar to the MT solution, in motility buffer) for $10 \mathrm{~min}$, and then washed with $50 \mu \mathrm{l}$ of motility buffer. Hereafter, $C_{S T}$ represents the concentration of the ST solution. Finally, the motility assay was initiated by applying $24 \mu \mathrm{l}$ of ATP solution (motility buffer supplemented with $5 \mathrm{mM} \mathrm{ATP}$ ). Solutions that had three different $C_{\mathrm{k}}$ values were prepared simultaneously, and ATP additions were delayed for $15 \mathrm{~min}$. To avoid drying, the flow cell was sealed with grease.

One concentration ( $C_{\mathrm{k}}, C_{\mathrm{ST}}$, or $C_{\mathrm{Tub}}$ ) was varied to investigate the effects on MT assembly formation, while the others were fixed as $C_{\mathrm{k}}=63 \mathrm{nM}, C_{\mathrm{ST}}=C_{\text {Tub }}$, and $C_{\text {Tub }}=24 \mathrm{nM}$. In the study of GMPCP-MTs, i.e., studying the effect of increased rigidity in MT, $C_{\text {Tub }}$ was kept at $240 \mathrm{nM}$; for comparative experiments, GTP-MTs were also used with $C_{\text {Tub }}=240 \mathrm{nM}$.

\subsection{Microscopic image capture}

For motility assays, the MT should be labeled with as little fluorescent probe as possible to retain activity. A highly sensitive camera is desirable to capture the weak fluorescence image of the MTs.

In our experiments, rhodamine-labeled MTs were illuminated with a $100 \mathrm{~W}$ mercury lamp and visualized by epifluorescence microscopy using a PlanApo $60 \times / 1.40$ objective. The

pg. 6 
images were captured using an EM-CCD camera (Cascade II, Nippon Roper) connected to a computer. To capture a field of view for more than several minutes, ND filters were inserted into the illuminating light path of the fluorescent microscope to avoid photo-bleaching of the rhodamine-labeled MTs. The images were captured using Image Pro Plus 5.1] software (Media Cybernetics). Although the inner surface of the cover glass was observed through the glass, the captured images corresponded to those observed from the reverse side of the flow cell.

\subsection{Image analysis for density measurements and motility analysis}

Fluorescence microscopy images of MTs and MT bundles captured $15 \mathrm{~min}$ and $4 \mathrm{~h}$ after (or before) ATP addition were analyzed using Image Pro Plus 5.1] or Image J 1.44f [ref. 41-43].

MT bundles were distinguished as segments that were obviously brighter than the other MTs. Small objects, whose lengths and widths were indistinguishable, were not counted. In cases where the MTs crossed each other, the lengths were measured as follows. When MTs overlapped at a point, they were measured independently. When they overlapped lengthwise in a segment, longer MTs were measured as a single piece, and the others were measured only in the non-overlapping sections. In the case of ring-shaped MT bundles, the circumferential lengths of the bundles were measured. For MTs that were partially invisible at the end of the viewing field, only the visible parts were measured. The total contour lengths of MTs per field of view $\left(135 \times 135 \mu \mathrm{m}^{2}\right)$ were measured manually and converted to the length per unit field. The total length of the MTs, including the MT bundles, per unit area was termed the "total sum length of the MTs $\left(L_{\text {total }}\right)$," and the total length of only the MT bundles per unit area was termed the "total bundle length" $\left(L_{\text {bundle }}\right)$. To calculate the individual length for the histogram and the average length, we excluded the MTs and MT bundles that were not entirely visible.

In this study, the mean velocities of the MT bundles were obtained from those showing sliding motion. MT bundles that did not show sliding motion were excluded from the calculation of the mean velocities. 


\section{Results and Discussion}

\subsection{Integration of the gliding MTs}

The schema of the procedure used to perform active self-organization (AcSA) of MT bundles is shown in Figure 1. AcSA was started by adding ATP to the system. Figure 2a shows a conceptual model of the AcSA system of MT bundles in which MTs are crosslinked by the ST-biotin interaction during the sliding motion on a kinesin-coated surface. This bundling event was confirmed by observing the sequential images of florescence microscopy driven by ATP hydrolysis (Figure 2b).

Although the bundling event was infrequent among the encountering MTs, the pattern of bundled MTs after several hours of AcSA were obviously different from the initial state (15 min after ATP addition) (Figure 3a). From this observation, the time point of $4 \mathrm{~h}$ after AcSA was considered as the matured phase for bundle formation, since bundle formation was confirmed by an increase in MT fluorescence intensity.

MTs with increased rigidity can be prepared by polymerizing tubulins with guanylyl-(a, ß)-methylene-diphosphonate (GMPCPP). Compared to the normal MTs, which were prepared by polymerizing with GTP and stabilizing with paclitaxel, the GMPCPP-polymerized MTs (GMPCPP-MTs) have ca. 2-fold higher flexural rigidity [ref. 40, 41]. Using GMPCPP-MTs, the MT bundles were successfully formed. In contrast to the serpentine shape of the MT bundles composed of paclitaxel-stabilized GTP-MTs, GMPCPP-MTs as well as their bundles were straight for several tens of micrometers (Figure 3d-f).

To clarify the effect of the initial conditions of AcSA on the size, amount, and motility of the MT bundles, we examined the concentration of the components (kinesin, ST, and MT) and rigidity of the MTs.

\subsection{Length and density of MT bundles}

\section{Effect of $C_{\mathrm{k}}$}

First, we studied the effect of $C_{\mathrm{k}}$ on AcSA when $C_{\text {ST }}(9.6 \mathrm{nM})$ and $C_{\text {Tub }}(9.6 \mathrm{nM})$ were kept constant. At $C_{k}$ values of $0.63,6.3$, and $63 \mathrm{nM}$, the density of the MTs ( $\left.L_{\text {total }}\right)$ before starting AcSA were 6.3, 9.1, and $13.2 \mathrm{~mm}^{-1}$, respectively, while the average lengths of the MTs were $6.0 \pm 4.8(n=177), 6.6 \pm 5.3(n=236)$, and $7.2 \pm 5.9 \mu \mathrm{m}(n=286)$, respectively (Figure $4 b$ ). The higher kinesin density enabled to bind longer MTs against the flow during 
preparation. After running AcSA, the amount of MTs and MT bundles was measured (Figure 4b). After $4 \mathrm{~h}$ of AcSA, $L_{\text {bundle }}$ at a $C_{\mathrm{k}}$ of $63 \mathrm{nM}$ was higher than that at a $C_{\mathrm{k}}$ of $6.3 \mathrm{nM}$, while no distinct trend was observed 15 min after ATP addition. At a $C_{k}$ of $0.63 \mathrm{nM}$, the MTs did not move and AcSA did not progress. After $4 \mathrm{~h}$ of ATP addition, the fraction of MT bundles increased to $46 \%$ and $39 \%$ at $C_{\mathrm{k}}$ values of 6.3 and $63 \mathrm{nM}$, respectively, although the fraction of MT bundles was only $3 \%$ at a $C_{\mathrm{k}}$ of $0.63 \mathrm{nM}$. These data indicate the significance of MT movement for AcSA. In comparison to the number of short MTs at a higher kinesin density ( $C_{\mathrm{k}}$ of $\left.63 \mathrm{nM}\right)$, a lower kinesin density ( $C_{\mathrm{k}}$ of $6.3 \mathrm{nM}$ ) could also lead to fewer short MTs at $4 \mathrm{~h}$, as shown in Figure $4 \mathrm{~b}$. Thus, to obtain greater amounts of motile MT bundles, a high $C_{\mathrm{k}}$ is required, although MT bundles can be formed even at a low $C_{k},(6.3 \mathrm{nM})$. Diehl et al. reported that multimerized kinesin on a glass surface shows higher MT-driving activity. Using multimerized kinesins with higher driving activity, MT bundles might be produced effectively [ref. 7].

\section{Effect of $C_{S T}$}

The average lengths of the MTs on the kinesins before starting AcSA were insensitive to $C_{S T}$ and ranged between 10 and $12 \mu \mathrm{m}$. The average number density of the MTs on the kinesins was approximately $2500 \mathrm{~mm}^{-2}$ at each $C_{S \mathrm{ST}}$. At a $C_{\mathrm{ST}}$ higher than $0.48 \mathrm{nM}$, the assembly of MTs of various shapes, such as linear, ring, and buckling (Figure 3c), was observed. By $4 \mathrm{~h}$ of AcSA, MTs were shorter than before the assay began. A similar phenomenon was reported earlier [ref. 44] and was explained on the basis of the "breakage" or "shrinkage" of the MTs moving on the kinesin-coated surface. With an increase in $C_{S T}$, the number of shortened MTs increased; most of the MTs and MT bundles were less than $10 \mu \mathrm{m}$ in length at an ST of $4800 \mathrm{nM}$. Thus, steric hindrance at the leading end of the MTs seems to cause "buckling" (Figure 3c). At a $C_{S T}$ higher than $48 \mathrm{nM}$, it was difficult for MT bundles longer than $25 \mu \mathrm{m}$ to form, presumably because of the shortening of the MTs. Meanwhile, the number of MT bundles containing the "buckling-shape" MTs increased with an increase in $C_{S T}$. The fraction of MT bundles ( $L_{\text {bundle }}$ ) decreased with time at $C_{S T}=0$, indicating that the MT bundles that were formed before starting AcSA were broken during the process (Figure 4d). At $4 \mathrm{~h}$ after ATP addition, the $L_{\text {bundle }}$ increased with an increase in $C_{S T}$ and almost plateaued above 480 nM. After $4 \mathrm{~h}$ of AcSA, the fraction of MT bundles increased with an increase in $C_{S T}$. The fraction of MT bundles was approximately $40 \%$ at ST concentrations higher than $480 \mathrm{nM}$, whereas it was only approximately $4 \%$ at a $C_{\text {ST }}$ of $0 \mathrm{nM}$. Thus, the higher $C_{\text {ST }}$ resulted in both a larger $L_{\text {bundle }}$ and a larger fraction of MT bundles; The longest MT bundles were observed at a $C_{\mathrm{ST}}$ of $\sim 48 \mathrm{nM}$.

\section{MT bundle formation: Effect of $C_{T u b}$}

pg. 9 
The $L_{\text {total }}$ values before starting AcSA varied from 2 to $175 \mathrm{~mm}^{-1}$ to change the $C_{\text {Tub }}$ from 1.92 to $240 \mathrm{nM}$. The average lengths of the MTs were also constant and ranged between 7.4 and $8.3 \mu \mathrm{m}$. Again, various shapes such as linear, ring, and buckling were observed at each $C_{\text {Tub }}$ after $4 \mathrm{~h}$ of AcSA. Shortening of the MTs was enhanced at a higher $C_{\text {Tub }}$ which suggests that entanglement of MTs leads to shortening of the MTs during AcSA. The average length of the MT bundles was insensitive to $C_{\text {Tub }}$ between 9.2 and $11.1 \mu \mathrm{m}$. However, at a $C_{\text {Tub }}$ of $240 \mathrm{nM}$, elongation of the MT bundles was observed, and the longest MT bundle was $\sim 50 \mu \mathrm{m}$. Prior to ATP addition, $L_{\text {bundle }}$ increased with an increase in $C_{\text {Tub }}$ (Figure $4 \mathrm{f}$, before AcSA). A decrease in $L_{\text {bundle }}$ with time was observed after the AcSA process ( $15 \mathrm{~min}$ and $4 \mathrm{~h}$ ) at $C_{\text {Tub }}$ values of 1.9 and $2.4 \mathrm{nM}$. At $C_{\text {Tub }}$ values of $48 \mathrm{nM} \mathrm{(15} \mathrm{min} \mathrm{and} 4 \mathrm{~h}$ ) and $240 \mathrm{nM}$ (15 $\mathrm{min}), L_{\text {bundle }}$ values were higher than those before AcSA, indicating that the MT bundle was produced by AcSA. However, at a $C_{\text {Tub }}$ of $240 \mathrm{nM}(4 \mathrm{~h}), L_{\text {bundle }}$ was shorter than that at before AcSA or at $15 \mathrm{~min}$. This result implies that the MT bundles were thickened because of their integration with an increase in the density of MT bundles. After $4 \mathrm{~h}$ of AcSA, the fraction of MT bundles increased with an increase in $C_{\text {Tub, }}$ which was also observed 15 min after ATP addition (Figure 4f, $15 \mathrm{~min}$ ). MT bundles formed prior to ATP addition seemed to be broken because the MT bundle fraction decreased at $C_{\text {Tub }}$ values of 1.9 and $9.6 \mathrm{nM}$ during AcSA. The formation of MT bundles can be confirmed based on two results. First, the fraction of MT bundles increased with time and $C_{\text {Tub }}$. Second, long MT bundles (up to $\sim 30 \mu \mathrm{m}$ ) were not observed before the AcSA process. Assuming that the decrease in the fraction of initial MTs was due to the consumption of MTs to thicken the MT bundles, the widths of the bundles were roughly estimated to be 1.5- and 3.1-fold of MTs at $C_{\text {Tub }}$ values of 48 and 240 nM, respectively.

\subsection{Rigidity of MTs on MT bundle formation}

Using rigid microtubules (GMPCPP-MTs), large, straight MT bundles were selectively obtained by AcSA. The average length of the GMPCPP-MTs before starting AcSA was $12.9 \pm$ $7.8 \mu \mathrm{m}(\mathrm{n}=352)$ on the kinesin-fixed surface, which is approximately the same length as that of the GTP-MTs $(11.0 \pm 8.3 \mu \mathrm{m}[\mathrm{n}=303])$. No ring-shaped GMPCPP-MT bundles were observed in the randomly selected view fields, which is in contrast to the case of GTP-MTs (Figure 3) where $\sim 4 \%$ of the MT bundles were ring-shaped. The average length of the GMPCPP-MT bundles at $4 \mathrm{~h}$ of AcSA was $26 \pm 19 \mu \mathrm{m}(n=56)$. The longest GMPCPP-MT bundle was $94 \mu \mathrm{m}$. $L_{\text {total }}$ of the GMPCPP-MTs before and after $4 \mathrm{~h}$ of AcSA were $144 \mathrm{~mm}^{-1}$ and $42 \mathrm{~mm}^{-1}$, respectively. Based on these observations, it was estimated that the GMPCPP-MT bundles were composed of approximately 3.4 GMPCPP-MTs. 


\subsection{Motility}

MT bundles as candidates for biomachines are of great interest due to their motility. We investigated the motion of MT bundles at various concentrations ( $C_{\mathrm{k}}, C_{\mathrm{ST}}$, or $\left.C_{\mathrm{Tub}}\right)$ and the rigidity of MTs in AcSA. In Figure 5a, motilities of the MTs and MT bundles after $4 \mathrm{~h}$ of AcSA at different $C_{\mathrm{k}}$ values are shown. Only $50 \%$ of the MTs and MT bundles were moving at a $C_{\mathrm{k}}$ of $6.3 \mathrm{nM}$, whereas $90 \%$ of these were still actively moving at a higher $C_{\mathrm{k}}(63 \mathrm{nM})$. Figure $5 \mathrm{~b}$ shows a study of the MTs and MT bundles at various $C_{S T}$ values. Although the presence of ST $(0.48-4.8 \mathrm{nM})$ had no influence on the motility of the MT bundles, $50 \%$ of these showed no sliding motion at a $C_{S T}$ of $48 \mathrm{nM}$, and only a few MT bundles moved at an ST concentration higher than $480 \mathrm{nM}$. MT bundle formation was not observed at a $C_{S T}$ of $0 \mathrm{nM}$ in the movie recorded in this assay. The differences in the motilities at various $C_{\text {Tub }}$ values are shown in Figure 5c. At a $C_{\text {Tub }}$ of $1.9 \mathrm{nM}, \mathrm{MT}$ bundles did not move. At $C_{\text {Tub }}$ values of 9.6 and $48 \mathrm{nM}$, $83 \%$ of the MT bundles were found to be motile. At a $C_{\text {Tub }}$ of $240 \mathrm{nM}$, only $17 \%$ of the MT bundles showed sliding motion. Moreover, 24\% showed fish-tailing motion with a "buckling" shape, whereas approximately $59 \%$ were static.

The use of GMPCPP-MTs led to higher motility: $94 \%$ of the GMPCPP-MT bundles were still actively moving and their linear shape was maintained, which was in contrast to the bundles formed with GTP-MTs (44\%, $n=27)$ in the control experiment. The average velocity of the GMPCPP-MT bundles was $0.162 \pm 0.040 \mu \mathrm{m} \mathrm{s}^{-1}(n=20)$ at $4 \mathrm{~h}$ of AcSA, which is the same as that for GMPCPP-MTs (0.165 $\pm 0.043 \mu \mathrm{m} \mathrm{s}^{-1}, n=38$, only single MTs) at the beginning (15 $\mathrm{min}$ ) of the process. The average velocities of the GTP-MTs (i.e., single MTs) at $15 \mathrm{~min}$ and their bundles at $4 \mathrm{~h}$ were $0.165 \pm 0.053 \mu \mathrm{m} \mathrm{s}^{-1}(n=53)$ and $0.189 \pm 0.048 \mu \mathrm{m}$ $\mathrm{s}^{-1}(n=12)$, respectively. Owing to the rigidity, the GMPCPPP-MTs and their MT bundles moved in a straighter trajectory than those of GTP-MTs, as previously discussed in detail [ref. 42].

Apart from the linear shape and motion of the GMPCPP-MT bundles, ring-shaped MT bundles were observed in AcSA with normal MTs (GTP-MTs). With the lapse of observation time, fewer and fewer MT bundles in translational motion were noted in the observation area since the motion was terminated at the walls of the flow cell; however, those in rotation remained in the observation area for more than $14 \mathrm{~h}$. Occasionally, MT aggregation was observed when the density of MTs was high enough to have frequent MT collisions. When the front of a sliding microtubule with a linear-shape collided with a rotating ring, it was spooled into the ring, as previously reported [ref. 24].

The rings showed constant rotational motion that was stabilized by the crosslinkage induced by streptavidin-biotin interactions. The angular velocity of the ring-shaped MT bundles depended on their size. The line velocity of the rotation remained almost constant, 
independent of the ring size. For example, the line velocity of counterclockwise rotation was found to be $28 \pm 14 \mathrm{~nm} \mathrm{~s}^{-1}$ (s.d.; $\mathrm{n}=5$ ), independent of the size, and was comparable to that of single MTs (28 $\left.\pm 23 \mathrm{~nm} \mathrm{~s}^{-1}, \mathrm{n}=19\right)$. We found only 5 ring-shaped MT bundles in the movies that could be used in the measurement of velocity and all of them were in the counterclockwise direction. These values are much lesser than those of single MTs without ST modification ( $360 \mathrm{~nm} \mathrm{~s}^{-1}$ ), presumably because of the consumption of ATP during $12 \mathrm{~h}$ of AcSA and ST labeling caused steric hindrance.

\subsection{Handedness of the structures and motions}

Since the ring-shaped MT bundles were rotating counterclockwise (CCW) or clockwise (CW), we investigated the distribution of the rotating directions. Surprisingly, the number of rings rotating $\mathrm{CCW}$ was always more than that of the rings rotating $\mathrm{CW}$ under various conditions. Figure 6 (right) shows the result of typical conditions $\left(C_{\mathrm{k}} 63 \mathrm{nM}, C_{\text {Tub }} 9.6 \mathrm{nM}, C_{\mathrm{ST}}\right.$ $\left.=C_{\text {Tub }}\right)$ for AcSA; a maximum ratio of CCW/CW of 222/16 ( $\left.\approx 14 / 1\right)$ was obtained at 240 nM MT and $63 \mathrm{nM}$ kinesin after $4 \mathrm{~h}$ of AcSA. To determine the cause of preferential CCW rotational motion, we observed the motion of single MTs without crosslinkers on a kinesin-coated surface. Results showed that among the 187 MTs observed, 173 showed translational motion and only 14 rotated. Of these 14 MTs, 6 were ring-shaped, 2 were spiral-shaped, 5 were arc-shaped, and 1 transformed its shape from spiral to arc; they all rotated CCW. Further observation of the MTs in rotation demonstrated that of the 37 MTs in rotation, 34 MTs rotated CCW and 3 rotated CW (Figure 6; left). In addition to this result, MTs are known to have a left- or right-handed supertwist in protofilament (PF) arrangement; PF is a tandem array of tubulin, configuring a hollow cylindrical structure of MT by closing up the parallel array of PFs [ref. 45-47]. Assuming that the handedness of MTs might induce biased motion, we determined the distribution of PF numbers in the MTs prepared for the AcSA by TEM observation [ref. 41]. Indeed, majority of MTs were 14 PFs that should have a left-handed supertwist arrangement, according to previous reports [ref. 45-47]. In a previous report, we proposed a mechanistic model to cause CCW rotational motion from a left-handed helical structure, since kinesin is known to follow the path of the PFs, i.e., the helical structure of the MTs. From these results and interpretations, the ring-shaped MT bundles could have handedness in the structure and the motion, harnessing the original structure of the MTs.

\subsection{The best conditions for motile MT bundles}

pg. 12 
To produce motile MT bundles with GTP-MTs, a higher $C_{\mathrm{k}}$ was favorable in the $C_{\mathrm{k}}$ range of 0-63 nM. Moreover, at the highest $C_{k}(63 \mathrm{nM})$, there was an optimum $C_{S T} / C_{\text {Tub }}$ of approximately $1 \mathrm{~mol} / \mathrm{mol}$ at which the AcSA process occurred effectively. We also found that a higher $C_{\text {Tub }}$ effectively produced a longer MT bundle, which was confirmed by the MT bundle fraction. However, there was an optimum $C_{\text {Tub }}$ of approximately $48 \mathrm{nM}$ that produced MT bundles with the ability to move.

To discuss the effects of $C_{S T}$ and $C_{\text {Tub }}$ on AcSA of MTs, the motile MT bundle length per unit area ( $L_{\mathrm{m} \text {-bundle }}$ ) at a $C_{\mathrm{k}}$ of $64 \mathrm{nM}$ was plotted against various $C_{\mathrm{ST}} / C_{\text {Tub }}$ ratios at a constant $C_{\text {Tub }}$ $(24 \mathrm{nM})$ and against various $C_{\text {Tub }}$ values at a constant $C_{\text {ST }} / C_{\text {Tub }}$ ratio $(\mathrm{mol} / \mathrm{mol})$ in a $3 \mathrm{D}$ diagram (Figure 7). In this case, $C_{\text {ST }}$ was normalized by $C_{\text {Tub }}$ as $C_{\text {ST }} / C_{\text {Tub }}(\mathrm{mol} / \mathrm{mol})$, and the motile MT-bundle length per unit area $\left(L_{m}\right.$-bundle $)$ was obtained by multiplying the percentage of

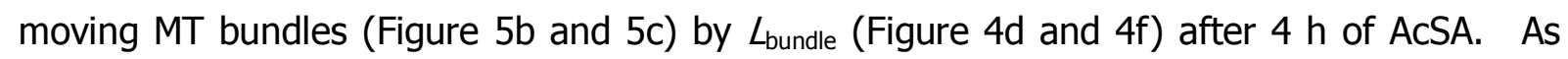
shown in the diagram, it was confirmed that an optimum $C_{\mathrm{ST}} / C_{\text {Tub }}$ and $C_{\text {Tub }}$ condition existed at around $1 \mathrm{~mol} / \mathrm{mol}$ (the labeling stoichiometry of biotins to MTs was 0.9 ) and $48 \mathrm{nM}$, respectively, and this facilitated the formation of large motile MT bundles.

For the case of GMPCPP-MTs, the bundles were much more motile than those of the GTP-MTs. From the highly motile property of these bundles, it was suggested that there could be a highly ordered structure with unipolarity.

\section{Conclusions}

In this paper, we summarized our recent studies on active self-organization of MTs. In our series of studies, it was found that motile MT assemblies that are linear or ring shaped could be obtained by tuning the initial conditions. Motor proteins have been proposed as the building blocks of ATP-fueled biomachines [ref. 7, 19, 24, 48]. For instance, MTs have been proposed as a molecular shuttle through control of the propelling direction with a micro-patterned wall [ref. 49]. Our large linear bundles can be applied to scale up this concept. In addition, we predict that our finding of preferential rotational motion in the CCW direction will provide new insights to create self-assembling biomachines with directional control. In the future, a combination with the technique that allows orientation of MTs in a 3D structure [ref. 50] or with the micro-fabrication technique, the application of this linear and highly motile MT bundles may increase the feasibility of developing ATP-fueled biomachines of nano, micro, and larger dimensions. Although many issues remain to be addressed in order to create versatile actuators with motor proteins, this study on MT bundle formation may provide insights into the design of biomachines with higher order structures. 


\section{Acknowledgements}

The plasmid construct of K560-GFP was generously provided by R. D. Vale and M. Tomishige. This study was financially supported by the Ministry of Education, Science, Sports and Culture, Japan (Grant-in-Aid for Specially Promoted Scientific Research).

\section{References}

1. Fenn W O 1923 J. Physiol. 58 175-203.

2. He Z-H, Bottinelli R, Pellegrino M A, Ferenczi M A and Reggiani C 2000 Biophys. J. 79 945-961.

3. Noji H, Yasuda R, Yoshida M and Kinosita Jr K 1997 Nature 386 299-302.

4. Yanagida T, Nakase M, Nishiyama K and Fumio O 1984 Nature 307 58-60.

5. Howard J, Hudspeth A J and Vale R D 1989 Nature 342 154-158.

6. Eickel V, Drummond D, Carter N, Lockhart A, Jones J K and Cross R 2004 FEBS Lett. 569 54-58.

7. Diehl M R, Zhang K, Lee H J and Tirrell D A 2006 Science 311 1468-1471.

8. Campas O, Leduc C, Bassereau P, Casademunt J, Joanny J-F and Prost J 2008 Biophys. J. 94 5009-5017.

9. Taba T, Edamatsu M, Toba S, Shibata K, Imafuku Y, Toyoshima Y Y, Tawada K and Yamada A 2008 Cell Motil. Cytoskeleton 65 816-826.

10. Vale R D 1999 Trends Cell Biol. 9 M38-42.

11. Limberis L and Stewart R J 2000 Nanotechnology 11 47-51.

12. Hess H and Vogel V 2001 Rev. Mol. Biotech. 82 67-85.

13. Böhm K J, Stracke R, Mühlig P and Unger E 2001 Nanotechnology 12 238-244.

14. Yokokawa R, Yoshida Y, Takeuchi S, Kon T and Fujita H 2006 Nanotechnology 17 289-294.

15. Kim T, Kao M-T, Meyhöfer E and Hasselbrink E F 2007 Nanotechnology 18 025101(9pp).

16. Suzuki H, Oiwa K, Yamada A, Sakakibara H, Nakayama H and Mashiko S 1995 Jpn. J. Appl. Phys. 34 3937-3941.

17. Korten T, Månsson A and Diez S 2010 Curr. Opin. Biotechnol. 21 477-488.

18. Agarwal A and Hess H 2010 Progress Polymer Sci. 35 252-277.

19. Kakugo A, Sugimoto S, Gong J P and Osada Y 2002 Adv. Mater. 14 1124-1126.

20. Kakugo A, Shikinaka K, Matsumoto K, Gong J P and Osada Y 2003 Bioconjugate Chem. 14 1185-1190.

21. Kwon H J, Kakugo A, Shikinaka K, Osada Y and Gong J P 2005 Biomacromol. 6 3005-3009.

22. Kwon H J, Tanaka Y, Kakugo A, Shikinaka K, Furukawa H, Osada Y and Gong J P 2006 Biochemistry 45 10313-10318.

23. Shikinaka K, Kwon H J, Kakugo A, Furukawa H, Osada Y, Gong J P, Aoyama Y, Nishioka H, Jinnai $\mathrm{H}$ and Okajima T 2008 Biomacromol. 9 537-542. 
24. Hess H, Clemmens J, Brunner C, Doot R, Luna S, Ernst K-H and Vogel V 2005 Nano. Lett. 5 629-633.

25. Shelanski M L, Gaskin F and Cantor C R 1973 Proc. Natl. Acad. Sci. USA. 70 765-768.

26. Weingarten M D, Suter M M, Littman D R and Kirschner M W 1974 Biochemistry 13 5529-5537.

27. Keating T J, Peloquin J G, Rodionov V I, Momcliovic D and Borisy G G 1997 Proc. Natl. Acad. Sci. USA. 94 5078-5083.

28. Castoldi M and Popov A V 2003 Protein Expr. Purif. 32 83-88.

29. Green N M 1990 Methods Enzymol. 184 51-67.

30. Hyman A, Drechsel D, Kellogg D, Salser S, Sawin K, Steffen P, Wordeman L and Mitchison T 1991 Methods Enzymol. 196 478-485.

31. Green N M 1970 Methods Enzymol. 18 418-424.

32. Peloquin J, Komarova Y and Borisy G 2005 Nat. Methods 2 299-303.

33. Vale R D, Reese T S and Sheetz M P 1985 Cell 42 39-50.

34. Svoboda K and Block S M 1994 Cell 77 773-794.

35. Kojima H, Muto E, Higuchi H and Yanagida T 1997 Biophys. J. 73 2012-2022.

36. Ludueña R F 1998 Int. Rev. Cytol. 178 207-75.

37. Case R B, Pierce D W, Hom-Booher N, Hart C L and Vale R D 1997 Cell 90 959-966.

38. Brown T B and Hancock W O 2002 Nano. Lett. 2, 1131-1135.

39. Vale R D, Coppin C M, Malik F, Kull F J and Milligan R A 1994 J. Biol. Chem. 269 23769-23775.

40. Mickey B and Howard J 1995 J. Cell Biol. 130 909-917.

41. Kawamura R, Kakugo A, Shikinaka K, Osada Y and Gong J P 2008 Biomacromol. 9 2277-2282.

42. Kawamura R, Kakugo A, Osada $Y$ and Gong J P 2010 Langmuir 26 533-537.

43. Kawamura R, Kakugo A, Osada Y and Gong J P 2010 Nanotechnology 21145603 (11pp).

44. Brunner C, Ernst K-H, Hess H and Vogel V 2004 Nanotechnology 15 S540-S548.

45. Chrétien D and Wade R H 1991 Biol. Cell 71 161-174.

46. Chrétien D, Kenney J M, Fuller S D and Wade R H 1996 Structure 4 1031-1040.

47. Chrétien D and Fuller S D 2000 J. Mol. Biol. 298 663-676.

48. Hess H, Bachand G D and Vogel V 2004 Chem. Eur. J. 10 2110-2116.

49. Brunner C, Wahnes C and Vogel V 2007 Lab. Chip 7 1263-71.

50. Kakugo K, Tamura Y, Shikinaka K, Yoshida M, Kawamura R, Furukawa H, Osada Y and Gong J P 2009 J. Am. Chem. Soc. 131 18089-18095. 


\section{Figures and Captions}

a

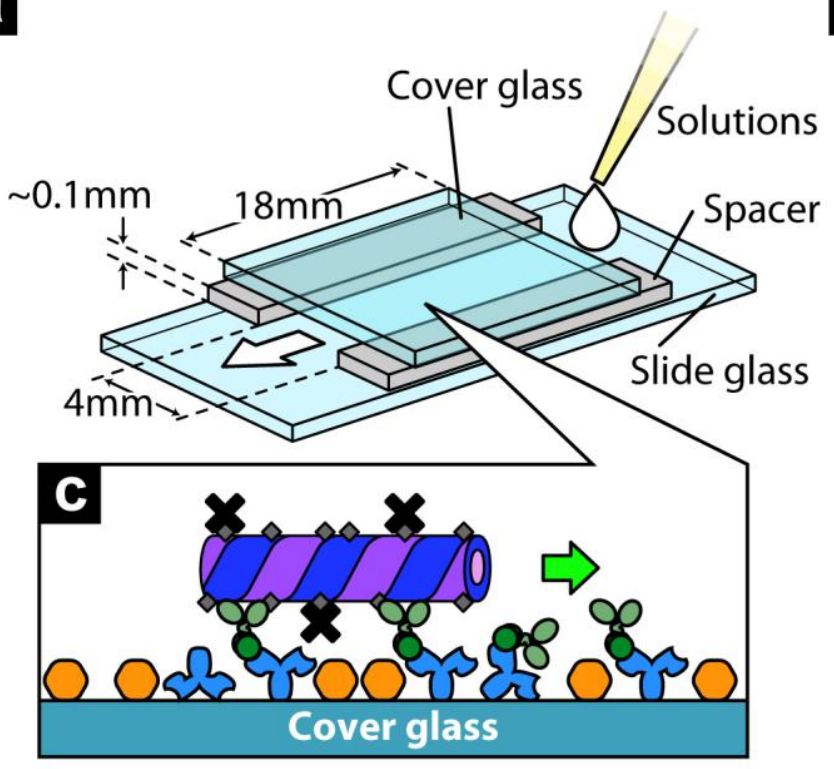

b $[$ Preparation of AcSA]

\begin{tabular}{|c|c|}
\hline & 1. Anti-GFP antibody \\
\hline & 2. Casein (blocking) \\
\hline & 3. Kinesin-GFP \\
\hline & 4. biotin-modified MT \\
\hline & 5. Streptavidin \\
\hline & Observation "before" \\
\hline & 6. ATP (starting AcSA) \\
\hline & Observation "15min" \\
\hline & Observation " $4 \mathrm{~h}$ "(or " $2 \mathrm{~h}$ ") \\
\hline
\end{tabular}

Figure 1: Schematic illustration of AcSA of MTs. (a) Dimensions of the flow cell. (b) Solutions introduced in the flow cell. The numbers represent the order in which each component solution was introduced into the flow cell; the unbound components at the surface of the flow cell were washed out before introducing the next solution or recording the observations. (c) Illustration of the MTs driven on kinesins coated on the glass surface. 

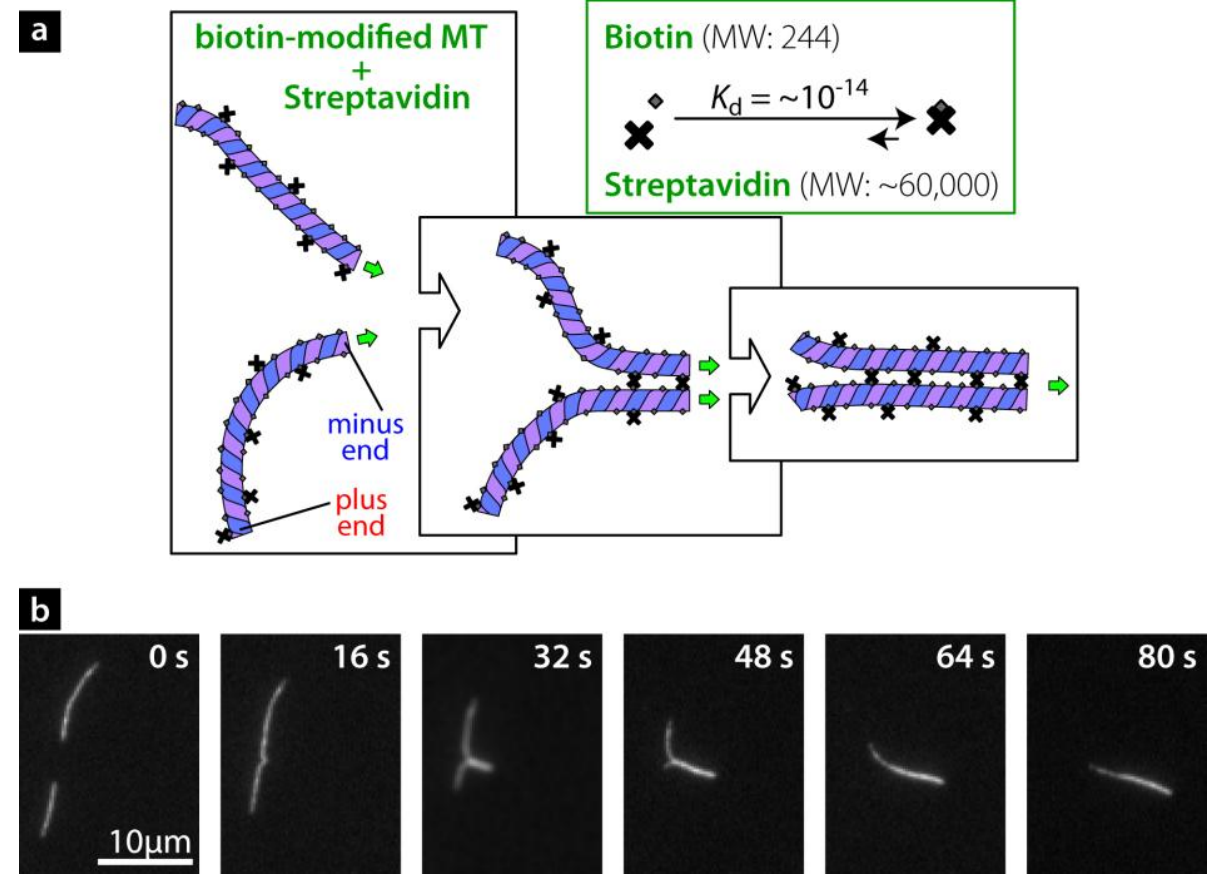

Figure 2: (a) Conceptual model of MT bundle formation. Two MTs are cross-linked by biotin-ST interactions. (b) Sequential images obtained under the fluorescent microscope view show MT bundle formation.

Active Self-assembly

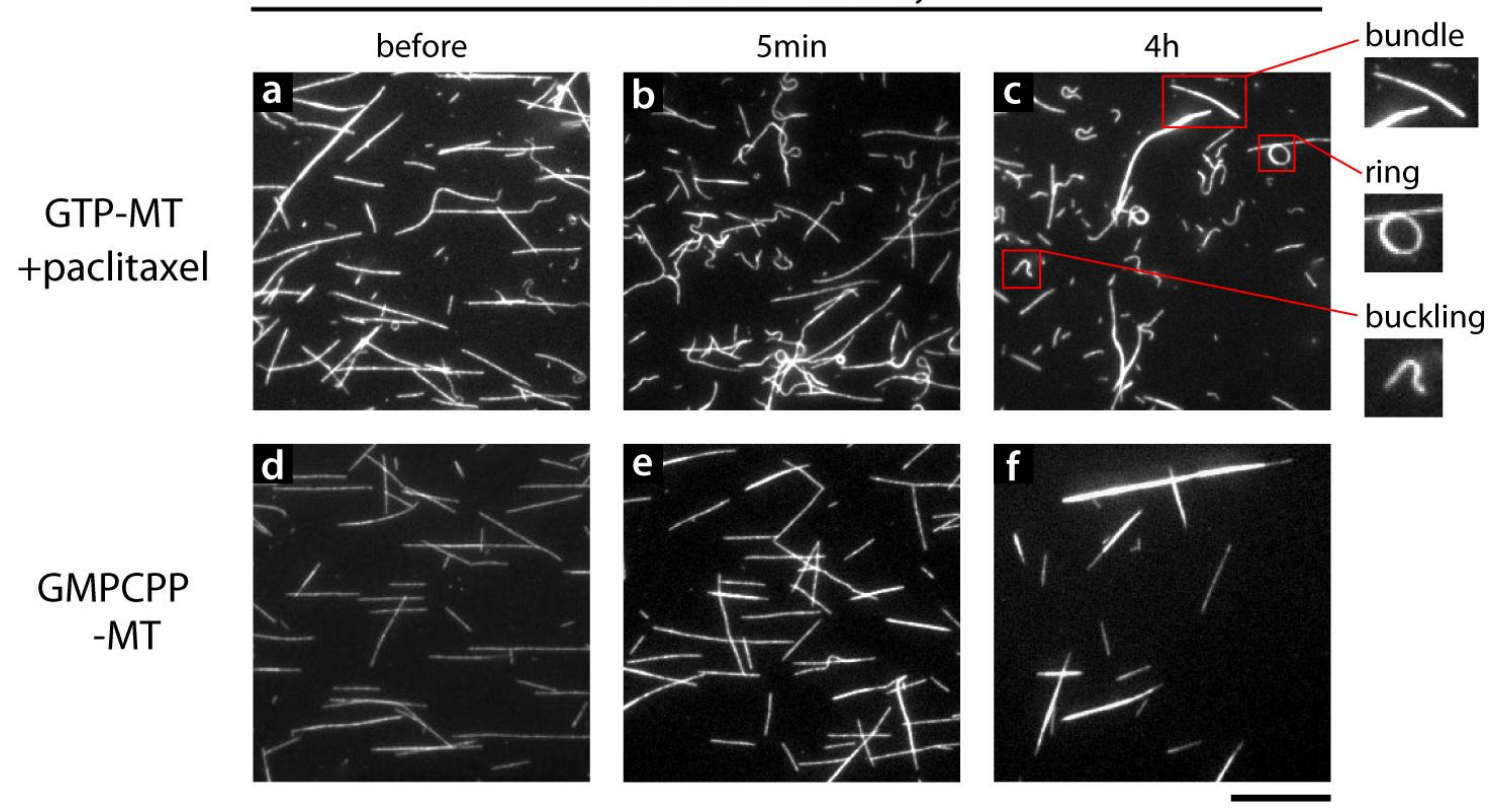

Figure 3

(a-c) GTP-MT: (a) before AcSA, (b) 15 min, (c) 4 h; (d-f) GMPCPP-MT: (d) before, (e) 15 min, (f) $4 \mathrm{~h}$ 

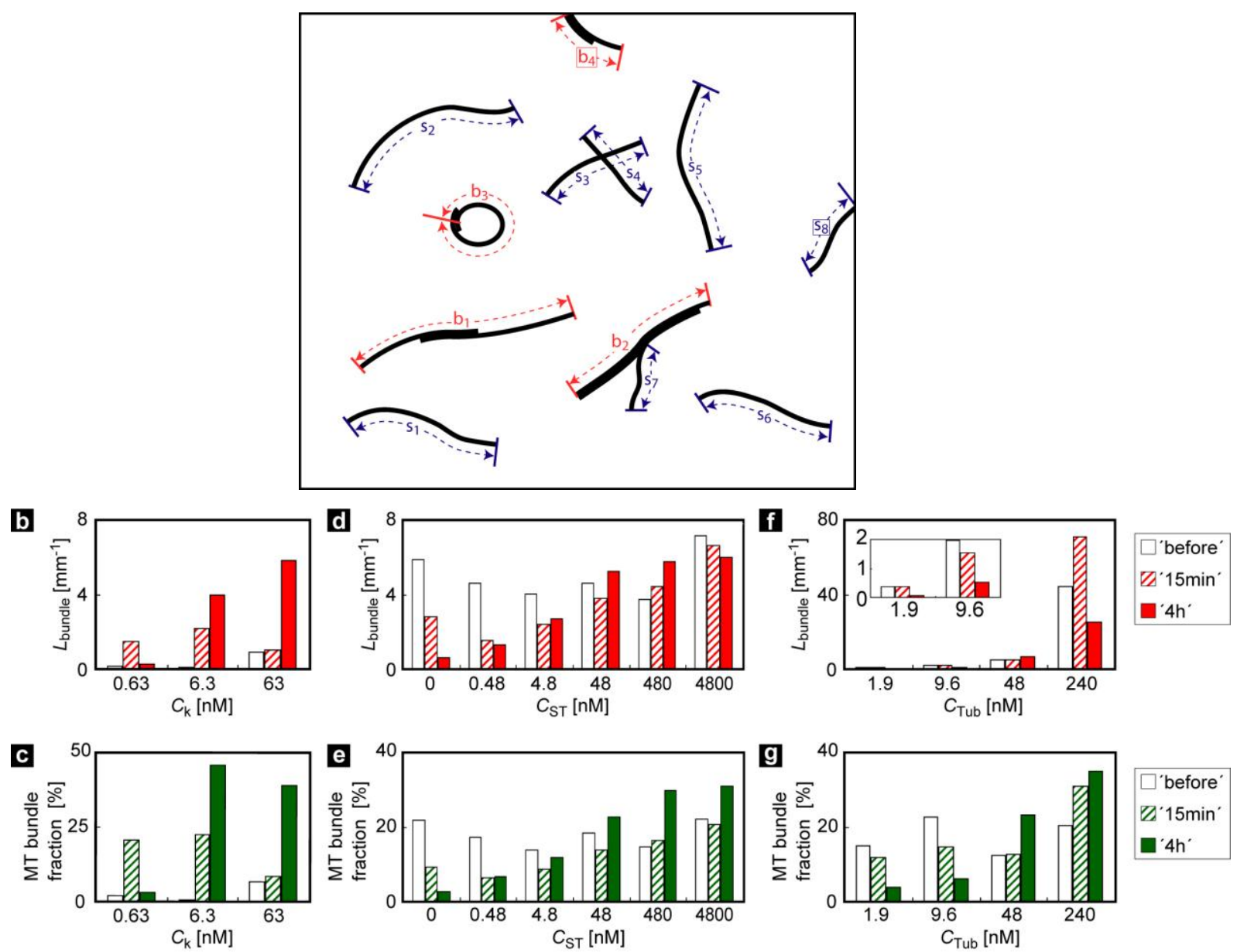

Figure 4: (a) Example of length measurement. The lengths of MTs and MT assemblies in the image are shown in blue and red, respectively. To evaluate the total sum lengths of MTs $\left(L_{\text {total }}: \mathrm{mm}^{-1}\right), \mathrm{s}_{1}-\mathrm{S}_{8}$ and $\mathrm{b}_{1}-\mathrm{b}_{4}$ were summed and divided by the area; $L_{\text {bundle }}$ was calculated by summing $b_{1}-b_{4}$. MT bundle fractions were calculated as $\left(L_{\text {bundle }} / L_{\text {total }}\right) \times 100$. Average lengths were calculated by excluding the object hidden by the frame $\left(s_{8}\right.$ and $\left.b_{4}\right)$.

(b)-(g) The results of length measurements. Each parameter of AcSA was varied as shown in the horizontal axis; $(\mathrm{b}, \mathrm{c}) C_{\mathrm{k},}(\mathrm{d}, \mathrm{e}) C_{\mathrm{ST}}$, and $(\mathrm{f}, \mathrm{g}) C_{\text {Tub. }}$ (b, d, f) shows the total sum lengths $\left(L_{\text {total }}\right)$ and $(c, e, g)$ shows the MT bundle fractions. For figures $(b)-(g)$, the original figures from ref 43 were modified with permission. 

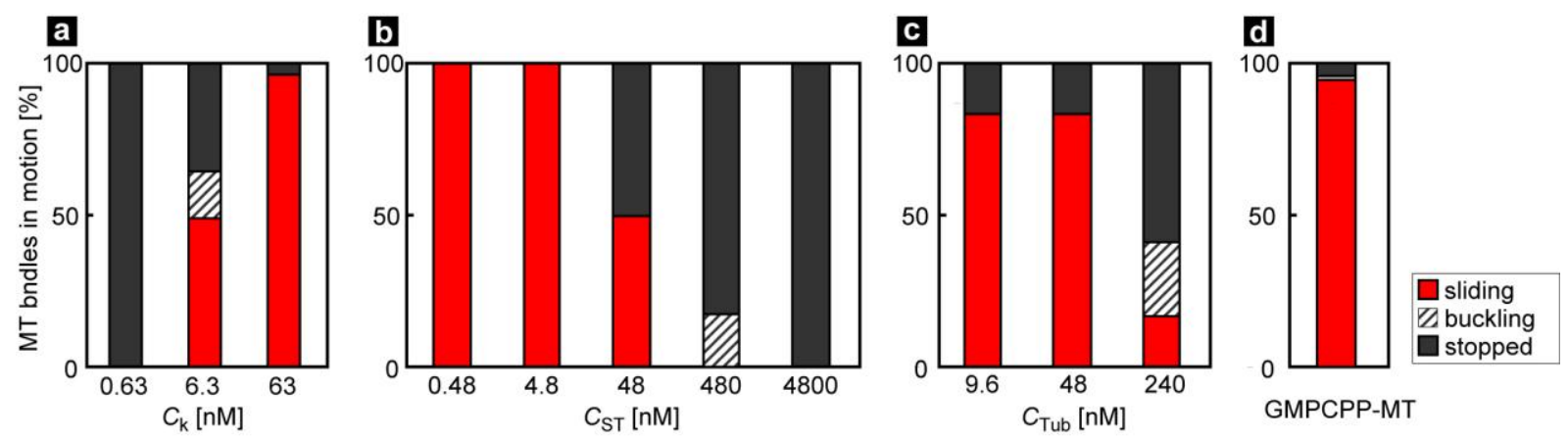

Figure 5: The percentage of moving MT bundles. Each parameter of AcSA was varied as shown in the horizontal axis; (a) $C_{\mathrm{k},}$ (b) $C_{\mathrm{ST}}$, (c) $C_{\text {Tub, }}$ and (d) with rigid MT. For figures (a)-(c), the original figures from ref 43 were modified with permission.

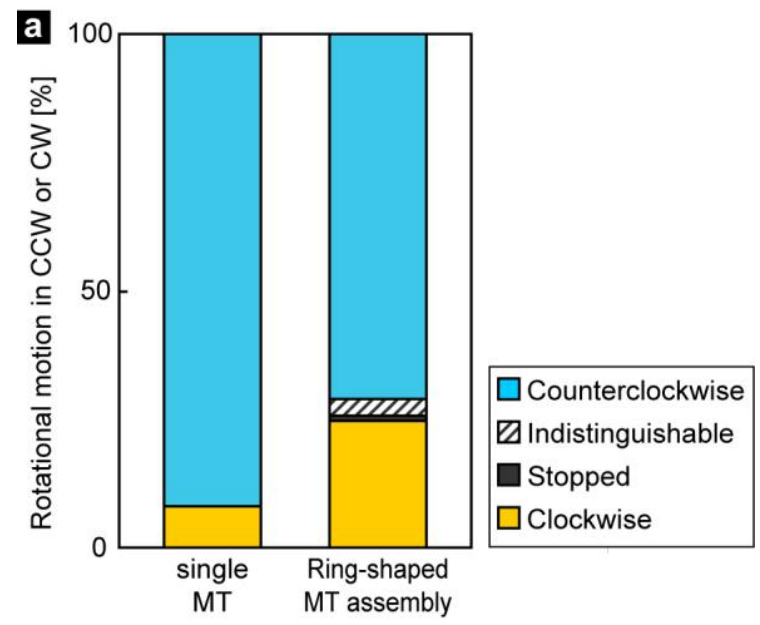

Figure 6: Distribution of the rotational motions in counterclockwise (CCW) or clockwise (CW) directions. $\quad C_{\mathrm{k}}$ and $C_{\text {Tub }}$ were kept $63 \mathrm{nM}$ and $9.6 \mathrm{nM}$, respectively, in the assays without a crosslinker (left) and with a crosslinker (right). The $C_{S T}$ was 0 and $9.6 \mathrm{nM}$ for (left) and (right), respectively. 


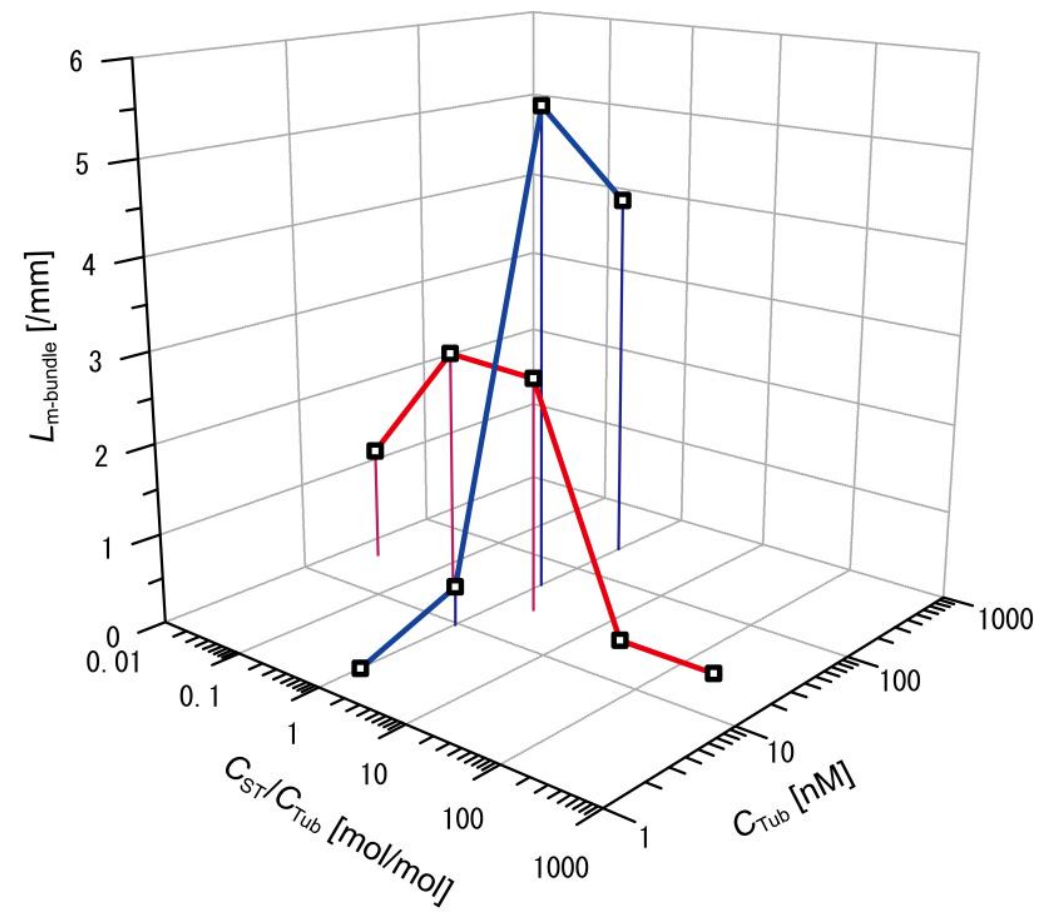

Figure 7: Estimation of the motile MT-bundle length per unit area at various $C_{\text {Tub }}$ and $C_{\text {ST }} / C_{\text {Tub }}$ ratios. Motile MT-bundle length per unit area ( $L_{\mathrm{m} \text {-bundle }}$ ) versus $C_{\text {Tub }}$ was calculated by multiplying $L_{\text {bundle }}$ (Figure $4 f$ ) and the percentage of moving MT bundles (Figure $5 c$ ), represented by the blue line. The red line represents $L_{\mathrm{m} \text {-bundle }}$ versus the $C_{\mathrm{ST}} / C_{\mathrm{Tub}}$ ratio; in this case, $C_{S T}$ was normalized by $C_{\text {Tub }}$ for comparison. Figure is taken from ref 43 with permission. 